\title{
80 Gbps NRZ-OOK Electro-Absorption Modulation of InP-on-Si DFB Laser Diodes
}

\author{
M. Shahin, J. Rahimi Vaskasi, J. Van Kerrebrouck, P. Ossieur, X. Yin, J. Bauwelinck, G. Roelkens, \\ and G. Morthier
}

\begin{abstract}
High-speed electro-absorption modulation of a heterogeneously integrated InP-on-Si DFB laser diode is demonstrated. The device has a 3-dB modulation bandwidth of 33 $\mathrm{GHz}$ and is used for the transmission of an $80 \mathrm{Gbps}$ NRZ-OOK signal over $2 \mathrm{~km}$ of NZ-DS fiber with BER below the hard-decision forward-error-correction threshold using feed-forward equalization.
\end{abstract}

Index Terms - InP-on-Si, distributed feedback lasers, electroabsorption modulation, silicon photonics.

\section{INTRODUCTION}

$\mathrm{S}$ ilicon photonics has been under extensive investigation by many researchers in recent years. The potential for large volume production at low cost makes this platform attractive for commercial applications. The field of silicon photonics offers the possibility of dense integration and the opportunity to use the well-developed CMOS electronics fabrication technology [1]. However, the main drawback of silicon lies in its indirect bandgap, which makes the implementation of light sources in this material quasi-impossible. Heterogeneous integration of III-V membranes on silicon, or silicon-oninsulator, is regarded as one of the few viable approaches to realize integrated sources.

One of the methods to heterogeneously integrate III-V semiconductors on silicon is die-to-wafer bonding [2]. In this method, a III-V semiconductor die is bonded epi-side-down on top of the silicon waveguide circuit. This bonding can be realized through molecular bonding or the use of an adhesive bonding agent such as divinylsiloxane-bis-benzocyclobutene (DVS-BCB). After the bonding, post-processing takes place for all III-V devices simultaneously. Another method of integration which is getting more attention nowadays is transfer printing [3], resulting in similar III-V-on-silicon device geometries.

The preferred light source for optical data-communication applications is the distributed-feedback (DFB) laser diode, thanks to its stable single mode behavior with large side mode

The authors acknowledge the Methusalem funding of the Flemish government, III-V Lab for supplying the epitaxial wafer, and the assistance of S. Verstuyft in the fabrication process.

M. Shahin, J. Rahimi Vaskasi, G. Roelkens and G. Morthier are with the Photonics Research Group, Department of Information Technology (INTEC), Ghent University-IMEC, and Center for Nanoand Biophotonics (NB-Photonics), Ghent University, Belgium. (Email: Mahmoud.Shahin@ugent.be, Javad.RahimiVaskasi@ugent.be, Gunther.Roelkens@ugent.be, Geert.Morthier@ugent.be). J. Van Kerrebrouck, P. Ossieur, X. Yin and J. Bauwelinck are with IDLab, Ghent University - imec, Ghent, Belgium. (E-mail: Joris.VanKerrebrouck@UGent.be, Peter.Ossieur@UGent.be, Xin.Yin@UGent.be; Johan.Bauwelinck@UGent.be). suppression. In the case of III-V-on-silicon devices, the Bragg grating can be defined in the silicon, using deep UV lithography, enabling their mass production.

A characteristic of great importance for data-communication is the maximum modulation speed of the transmitters. In recent years, several state-of-the-art results have been reported for both monolithic InP devices and heterogeneously integrated InP-on-Si devices. These were realized using different modulation schemes: non-return-to-zero on-off-keying (NRZOOK), 4-level pulse amplitude modulation (PAM-4), and electrical duobinary (EDB). NRZ-OOK is the simplest format with two-level modulation. It requires the highest bandwidth of the components but there are no stringent requirements on the linearity of the electronics and optics. It also provides the highest receiver sensitivity. A 100 Gbps NRZ GeSi electroabsorption modulator implemented on a silicon photonic platform was shown in [4].

For III-V-on-silicon devices specifically, 56 Gbps NRZOOK transmission over an optical link using direct modulation of an InP-on-Si DFB laser was shown in [5]. Electro-absorption modulation of the tapers, coupling the light from the III-V to the SOI, has been demonstrated as well. 56 Gbps NRZ was demonstrated from both sides of the laser [6]. For that purpose, lasers with emission wavelength close to the bandgap wavelength are selected in which the tapers are electrically isolated and reverse biased.

PAM-4 is a 4-level modulation scheme providing higher spectral efficiency. This allows doubling the bitrate for the same bandwidth, at the expense of lowering the modulation amplitude, and increasing the linearity and signal-to-noise requirements on electronic circuits and photonic devices. In [7], an InP distributed Bragg reflector (DBR) laser with a modulation bandwidth of $55 \mathrm{GHz}$ was reported. The laser was used for the generation of 112 Gbps PAM-4. Furthermore, an InP-based double-side electro-absorption-modulated DFB laser has been used for 2 x 56 Gbps PAM-4 [8]. For III-V-on-silicon devices, electro-absorption-modulated III-V-on-silicon DFB lasers have been used for 25 Gbaud PAM-4 [6], as well as 100 Gbps EDB in [9].

Although the bandwidth of directly modulated DFB lasers can be relatively high [7], modulation at high frequencies often comes with a large chirp. Electro-absorption modulation suffers less from this problem and its bandwidth is limited only by the $\mathrm{RC}$-constant of the modulator (where $\mathrm{C}$ is typically the junction capacitance and $\mathrm{R}$ is determined by the series resistance and output impedance of the driver). As the junction capacitance decreases with decreasing width and length of the EA modulator, a shorter and thinner EAM will result in a higher 
modulation bandwidth. However, a too short EAM would limit the extinction ratio and a too narrow device would limit the power handling, so a compromise must be sought. In this paper, we report on the NRZ-OOK electro-absorption modulation of a heterogeneously integrated III-V-on-silicon DFB laser at 80 Gbps. In the next section, we discuss the design and fabrication of the externally modulated laser diode (EML). We use adhesive bonding to heterogeneously integrate the InP epitaxial layer stack on the silicon waveguide circuit. We discuss how the design differs from the one reported in [5]. Next, we present the static measurement results of the EML as well as the small signal and large signal dynamic measurements. Using FeedForward Equalization (FFE), NRZ-OOK data transmission is verified at bitrates of $80 \mathrm{Gbps}$. The bit-error-rate (BER) is substantially below the 7\% hard-decision forward-errorcorrection (HD-FEC) threshold. All the results are shown for back-to-back configuration as well as after propagation over 2 $\mathrm{km}$ of non-zero dispersion-shifted-fiber (NZ-DSF).

\section{DESIGN AND FABRICATION}

The top-view and 3D-view of the laser structure are shown in Fig. 1. The taper structure used for coupling the light from the III-V-on-silicon DFB laser to the silicon waveguide is used as an EAM. The device is similar to that of [5], but with each taper section cut in two halves. Therefore, the device now consists of 5 sections: an active section in the middle, and two EAM sections on each end.

The sections are electrically isolated from each other during fabrication, by wet-etching the top $\mathrm{p}$-InGaAs contact layer, and dry-etching around $200 \mathrm{~nm}$ of the p-InP layer. The length of the etched region was $30 \mu \mathrm{m}$ instead of $7 \mu \mathrm{m}$ in [5]. A high electrical isolation can be achieved by making the isolation region longer, rather than deeper. Deep etching to isolate the layers can affect the optical mode, and reduce the output power. Isolation allows individual control over the bias in different sections. High resistance between the sections minimizes leakage current from one section to the other. In our laser, the resistance between the EAM and the laser section is $6 \mathrm{k} \Omega$.
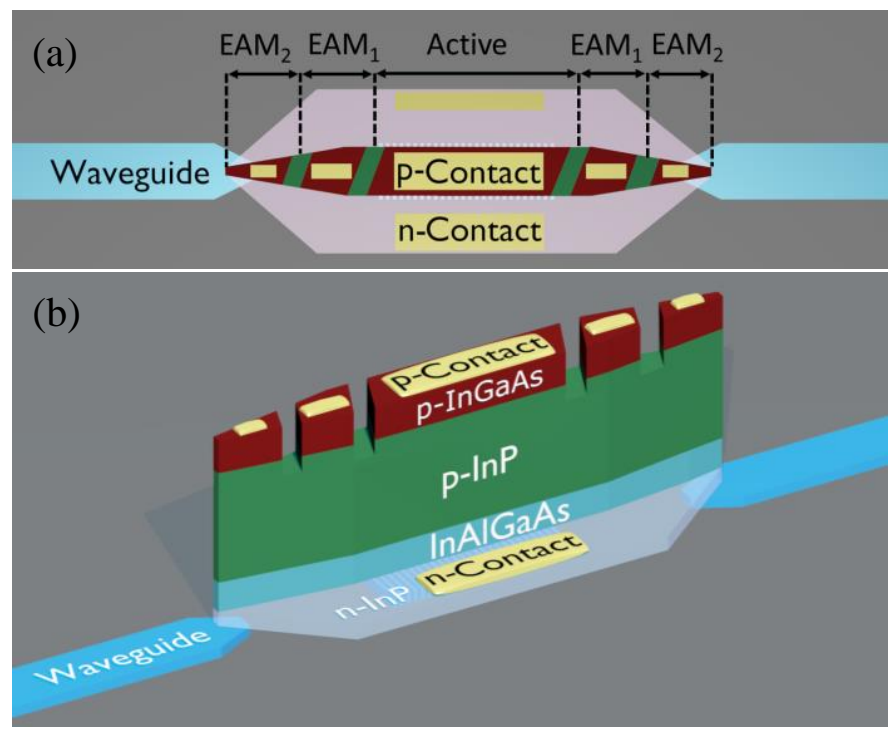

Figure 1. (a) Top-view and (b) 3D-view of the fabricated InP-on-Si EML showing the III-V-on-silicon DFB and tapers used as EAM.
The active laser section is $500 \mu \mathrm{m}$ long and $2 \mu \mathrm{m}$ wide. The EAM sections are each $50 \mu \mathrm{m}$ long. The full taper length is around $150 \mu \mathrm{m}$ long. The width of the taper goes from $2 \mu \mathrm{m}$ down to $0.7 \mu \mathrm{m}$.

In principle, either of the EAM sections can be used to modulate the light. However, in the first EAM section, the light is confined more to the III-V waveguide, while in the second EAM section the light is confined more to the silicon waveguide. Therefore, more light will be absorbed when reverse biasing the first EAM section, and a higher extinction ratio is expected. In this paper, we focus on modulating the first EAM section, while not pumping the second EAM section.

For ease of fabrication, the EAM epitaxial layer structure is the same as the laser epitaxial structure. It consists of 2 InGaAsP separate confinement heterostructure $(\mathrm{SCH})$ layers (50 nm thick, PL wavelength of $1170 \mathrm{~nm}$ ) and 8 InAlGaAs quantum wells ( $8 \mathrm{~nm}$ thick, PL wavelength $1550 \mathrm{~nm}$ ) separated by 9 InAlGaAs barriers (10 nm thick, PL wavelength of 1100 $\mathrm{nm})$. The thicknesses of the n-InP, $\mathrm{p}$-InP and p-InGaAs layers are $200 \mathrm{~nm}, 1850 \mathrm{~nm}$ and $220 \mathrm{~nm}$, respectively. If the lasing wavelength is close enough to the bandgap wavelength, reverse biasing of the EAM section will result in a significant extinction with low insertion loss. The InP epitaxial structure is bonded on a $400 \mathrm{~nm}$ thick Si waveguide ( $3.5 \mu \mathrm{m}$ wide) that has a $500 \mu \mathrm{m}$ long DFB grating etched $190 \mathrm{~nm}$ deep, with a period of $248 \mathrm{~nm}$ and duty cycle of $50 \%$. A quarter-wave shift is located in the center of the grating.

The fabrication starts with bonding the III-V die on top of the silicon waveguide structures. After substrate removal, the top p-InGaAs contact layer is dry-etched, followed by wet-etching of the p-InP, InAlGaAs and n-InP layers. Surface oxides on sidewalls of the InAlGaAs quantum wells and barriers are removed by repeated immersion in $\mathrm{H}_{2} \mathrm{SO}_{4}: \mathrm{H}_{2} \mathrm{O}_{2}: \mathrm{H}_{2} \mathrm{O}(1: 1: 20)$ and BHF: $\mathrm{H}_{2} \mathrm{O}$ (1:10). Then, the lasers are immediately passivated using low temperature $\mathrm{Si}_{3} \mathrm{~N}_{4}$, high temperature $\mathrm{Si}_{3} \mathrm{~N}_{4}$, and finally DVS-BCB. After etching back the DVS$\mathrm{BCB}$, the isolation etch is done, followed by metallization. The detailed fabrication steps are reported in [10].

\section{Characterization}

\section{Static characteristics}

The stage temperature was fixed at $15^{\circ} \mathrm{C}$. The output light is coupled out of the chip by means of a grating coupler, of which the coupling efficiency is $-6 \mathrm{~dB}$. The reported optical power $\mathrm{P}_{\text {out }}$ throughout the paper is the power in the $\mathrm{Si}$ waveguide (i.e. excluding the losses in the grating coupler). The threshold current $\mathrm{I}_{\mathrm{th}}$ is around $30 \mathrm{~mA}$. Fig. 2 shows the spectrum for $\mathrm{I}_{\text {active }}$ $=50 \mathrm{~mA}$ and $\mathrm{V}_{\text {EAM }}=0 \mathrm{~V}$. The lasing peak is at $1570.6 \mathrm{~nm}$ with $P_{\text {out }}=-4.4 \mathrm{dBm}$, as shown in Fig. 2. To characterize the EAM, $\mathrm{I}_{\text {active }}$ is fixed at $50 \mathrm{~mA}$, and $\mathrm{P}_{\text {out }}$ is measured at different $\mathrm{V}_{\mathrm{EAM}}$, as shown in Fig. 3. Between $\mathrm{V}_{\mathrm{EAM}}=0 \mathrm{~V}$ and $-1 \mathrm{~V}$, there is around $15 \mathrm{~dB}$ extinction.

\section{Small signal modulation}

A small signal modulation experiment is performed using the setup shown in Fig. 4 (a). An Agilent N5247A Vector Network Analyzer (VNA) is used to provide electrical signals up to 67 $\mathrm{GHz}$. The signal is superimposed on a negative bias, for reverse 
Optical Power dBm

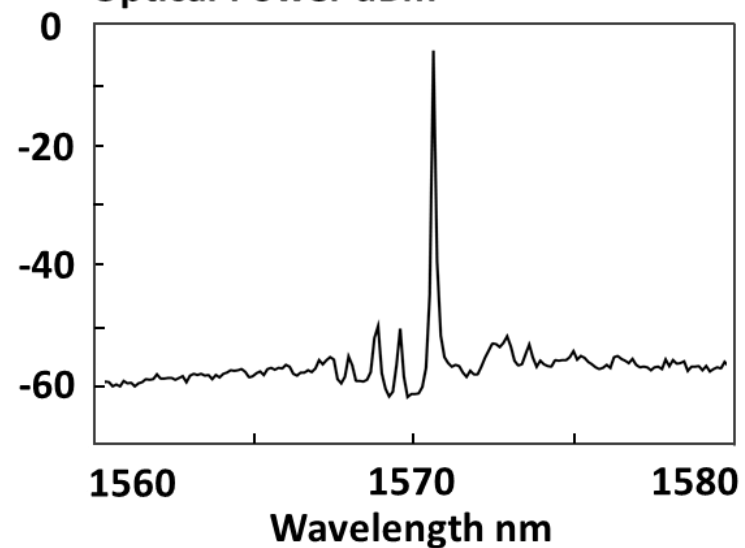

Figure 2. The spectrum of the laser showing single mode operation at 1570.6 $\mathrm{nm}$ for $\mathrm{I}_{\text {active }}=50 \mathrm{~mA}$, and $\mathrm{V}_{\mathrm{EAM}}=0 \mathrm{~V}$.

\section{Optical Power dBm}

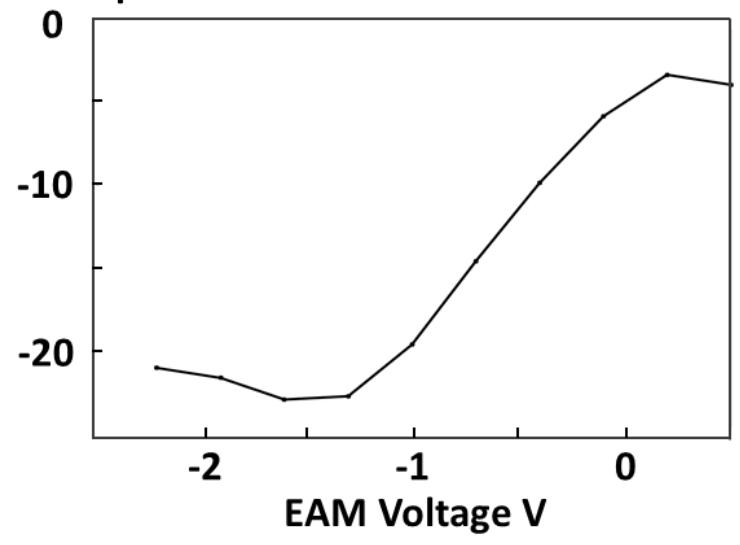

Figure 3. Optical output power $P_{\text {opt }}$ vs. EAM bias $V_{\text {EAM }}$ for $I_{\text {active }}=50 \mathrm{~mA}$.

biasing the EAM, using a $45 \mathrm{GHz}$ bias tee. The signal is then used to modulate the EAM, while biasing the DFB laser diode at $50 \mathrm{~mA}$ using a Keithley current source. Due to the losses in the optical link, i.e. from the grating coupler and to accommodate the fact that no trans-impedance amplifier was used in the experiment, the signal is boosted using an Optolink L-band Erbium Doped Fiber Amplifier (EDFA). The output is fed to a Discovery DSC-10H photo-detector with a bandwidth of $43 \mathrm{GHz}$. Finally, the output electrical signal is fed back to the VNA.

Fig. 5 shows the small signal $S_{21}$ parameter of the device. The measured 3-dB modulation bandwidth of the device was found to be around $33 \mathrm{GHz}$ at $\mathrm{I}_{\text {active }}=55 \mathrm{~mA}$ and $\mathrm{V}_{\mathrm{EAM}}=-0.8 \mathrm{~V}$. This is an improvement of around $30 \%$ compared to the $25 \mathrm{GHz}$ bandwidth reported in [5].

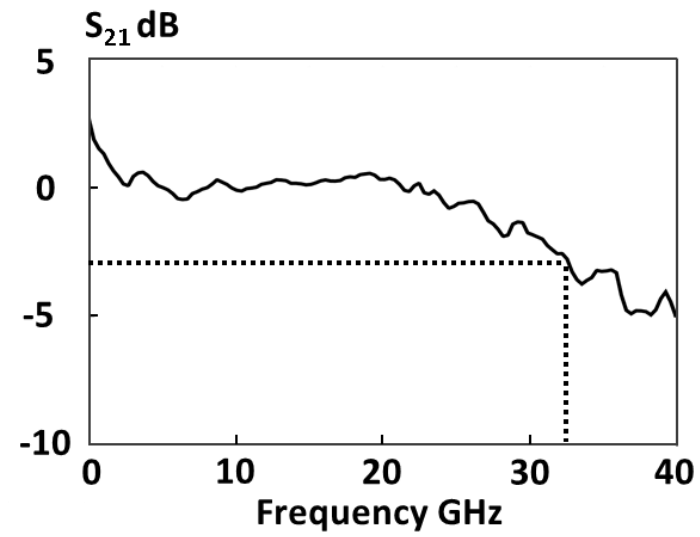

Figure 5. Small signal response showing nearly $33 \mathrm{GHz} 3-\mathrm{dB}$ modulation bandwidth at $\mathrm{I}_{\text {active }}=55 \mathrm{~mA}$ and $\mathrm{V}_{\mathrm{R}}=-0.8 \mathrm{~V}$.

\section{Large signal modulation}

Data transmission experiments were performed to investigate the large signal modulation performance of the EML. To do this, the setup in Fig. 4 (b) is constructed. An electrical signal is generated with a Keysight M8196A Arbitrary Waveform Generator (AWG). This electrical signal is amplified by a $50 \mathrm{GHz}$ RF electrical amplifier (SHF-S807). Similarly to the small signal measurement, the data signal is superimposed on a negative bias voltage.

An L-band EDFA is used to boost the optical signal. The amplified spontaneous emission from that EDFA is filtered out using a variable-bandwidth optical tunable filter (Santec OTF-350). The bandwidth of the filter was set to $1 \mathrm{~nm}$. The resulting optical signal is fed to the photodetector, and finally, the output electrical signal is fed to a Keysight DSAZ634A Real-Time Oscilloscope.

A raised-cosine filter with $\alpha=0.1$ is used to shape the transmitted pulse. Using the built-in channel de-embedding function of the AWG, transmission of 50 and 80 Gbps NRZ Pseudorandom-Binary-Sequence (PRBS) with a word length of $2^{7}-1$ was verified, as shown in Fig. 6. Fig. $6(a, c)$ show the eye diagram for back-to-back configuration, and Fig. 6(b, d) show the eye diagrams after transmission over a $2 \mathrm{~km}$ long NZ-DSF link.

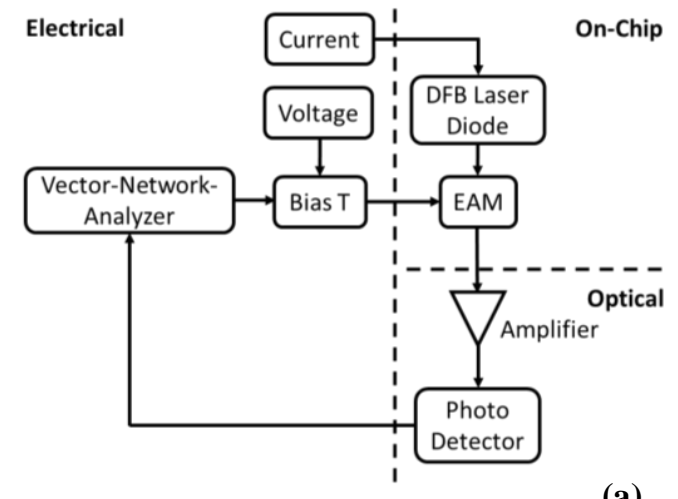

(a)

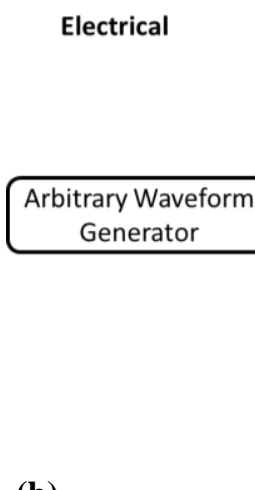

(b)

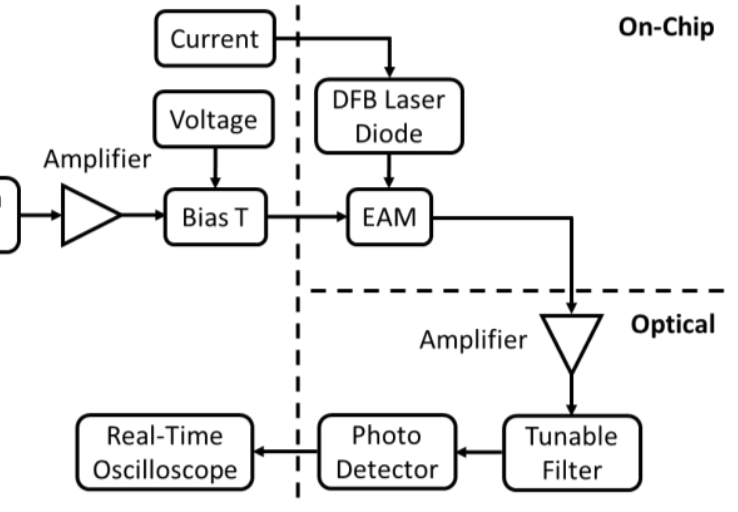

Figure 4. Block diagram of the measurement setup used for (a) small signal modulation and (b) data transmission experiments. 
The voltage swing applied to the EAM is about $1.8 \mathrm{~V}_{\mathrm{pp}}$. The device was operating at $I_{\text {active }}=49 \mathrm{~mA}$ and $\mathrm{V}_{\mathrm{EAM}}=-0.7 \mathrm{~V}$. The optical bandwidth of the optical tunable filter was $1 \mathrm{~nm}$. The received optical power was $5 \mathrm{dBm}$ (note that no transimpedance amplifier was present at the receiver). The recorded BER was around $1.5 \cdot 10^{-4}$, which is below the KP4FEC threshold.

Measurement of the BER vs. received power for this transmitter is shown in Fig. 7. To obtain different power levels at the receiver side and their BER, an optical attenuator is placed before the photo-detector. 80 Gbps PRBS transmission with a word length of $\left(2^{7}-1\right)$ with a BER lower than the $7 \%$ HD-FEC limit is possible for a received power less than 0 $\mathrm{dBm}$. It is observed that increasing the word length to $2^{15}$ increases the BER. This can be explained by looking back at the small signal modulation response in Fig. 5, in which the low frequency response has a $2 \mathrm{~dB}$ roll-off. This low frequency part becomes significant when bit combinations with low frequencies (many consecutive 1's or 0 's) become more probable.

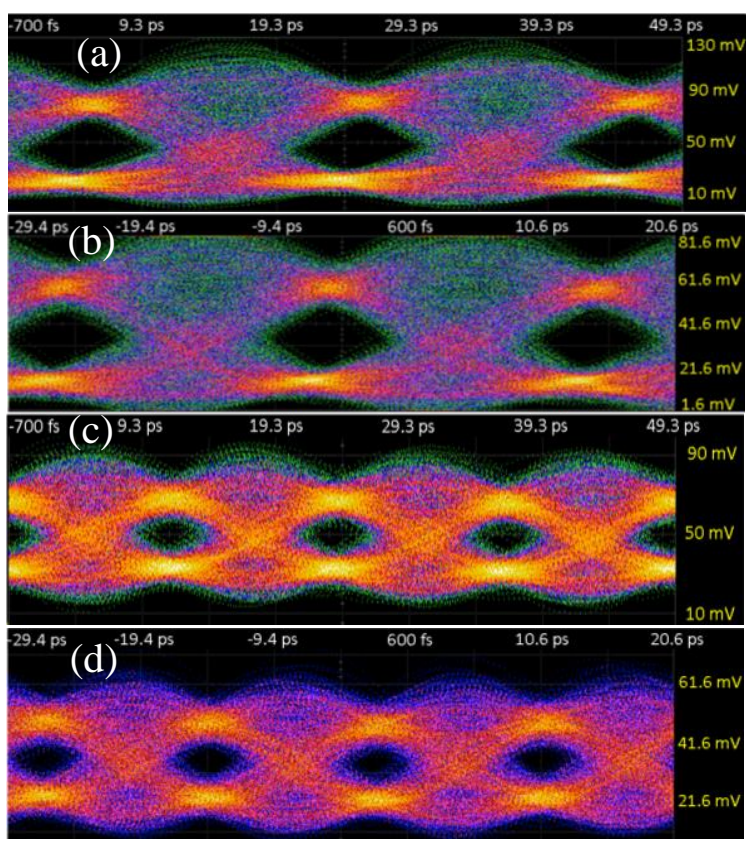

Figure 6. Sub-HD-FEC transmission at $50 \mathrm{Gbps}$ and $80 \mathrm{Gbps}(\mathrm{a}, \mathrm{c})$ in the back-to-back configuration, and (b, d) with a $2 \mathrm{~km} \mathrm{NZ-DSF} \mathrm{link,} \mathrm{for} \mathrm{a} \mathrm{pattern}$ length of $2^{7}-1$.

\section{CONCLUSIONS}

High-speed electro-absorption modulation of InP-on-Si DFB laser diodes is demonstrated. $33 \mathrm{GHz} 3-\mathrm{dB}$ modulation bandwidth and transmission of an 80 Gbps NRZ-OOK signal is shown for both a back-to-back configuration and for a $2 \mathrm{~km}$ long NZ-DSF link with a BER below the 7\% HD-FEC threshold using feed-forward equalization.

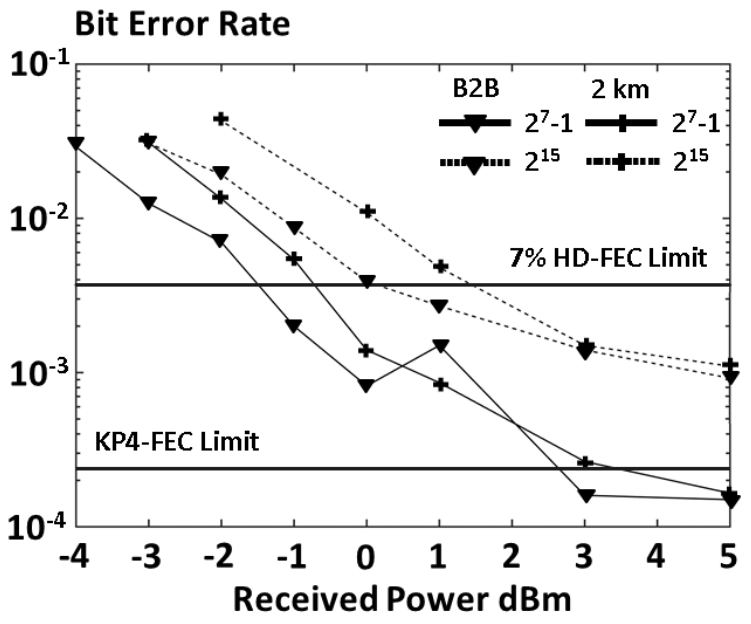

Figure 7. Bit-error-rate vs. received power showing sub-HD-FEC operation for $80 \mathrm{Gbps}$ for a received power below $0 \mathrm{dBm}$.

\section{REFERENCES}

[1] R. Soref, "The past, present, and future of silicon photonics," IEEE Journal of selected topics in quantum electronics 12(6), pp. 1678-1687, (2006).

[2] S. Keyvaninia et al., "Ultra-thin DVS-BCB adhesive bonding of III-V wafers, dies and multiple dies to a patterned silicon-on-insulator substrate," Optical Materials Express, 3(1), pp. 35-46, (2012).

[3] J. Zhang et al., "Transfer-printing-based integration of a IIIV-on-silicon distributed feedback laser," Optics Express, 26(7), pp. 8821-8830, (2018).

[4] J. Verbist et al., "Real-time $100 \mathrm{~Gb} / \mathrm{s} \mathrm{NRZ} \mathrm{and} \mathrm{EDB}$ transmission with a GeSi electro-absorption modulator for short-reach optical interconnects," Journal of Lightwave Technology, 36(1), p.90-96, (2018).

[5] A. Abbasi et al., "Direct and electro-absorption modulation of a III-V-on-silicon DFB laser at 56 Gbps," IEEE Journal of Selected Topics in Quantum Electronics, 23(6), pp. 1501307, (2017).

[6] A. Abbasi et al., "III-V-on-Silicon C-band High-Speed Electro-Absorption Modulated DFB Laser," Journal of Lightwave Technology, 36(2), pp.252-257, (2018).

[7] Y. Matsui et al., " $55 \mathrm{GHz}$ Bandwidth Distributed Reflector Laser," Journal of lightwave technology, 35(3), pp. 397-403, (2017).

[8] M. Theurer et al., " $2 \times 56$ GB/s From a Double Side Electroabsorption Modulated DFB Laser and Application in Novel Optical PAM4 Generation," Journal of lightwave technology, 35(4), pp. 706-710, (2017).

[9] A. Abbasi et al., "100-Gb/s Electro-Absorptive Duobinary Modulation of an InP-on-Si DFB Laser," IEEE Photonics Technology Letters, 30(12), pp. 1095-1098, (2018).

[10] G. Roelkens et al., "III-V-on-Silicon Photonic Devices for Optical Communication and Sensing," Photonics (3), pp. 9691004, (2015). 